\title{
Enantiopure Supramolecular Motifs of Self-assembled Diamine-based Chiral Molecules on $\mathrm{Cu}(\mathbf{1 0 0})$
}

\author{
Rogger Palacios-Rivera, ${ }^{1}$ Esther Barrena, ${ }^{* 1}$ Jordi Faraudo, ${ }^{1}$ Pierluigi Gargiani, ${ }^{2}$ Miguel \\ Angel Niño, ${ }^{3}$ Dimitri Arvanitis, ${ }^{4}$ Iwona Kowalik, ${ }^{5}$ Juan José de Miguel $^{6}$ and Carmen \\ Ocal $^{* 1}$
}

${ }^{1}$ Institut de Ciència de Materials de Barcelona (ICMAB-CSIC), Campus UAB, Bellaterra, E-08193, Barcelona, Spain.

${ }^{2}$ ALBA Synchrotron Light Source, Cerdanyola del Vallès, E-08290, Barcelona, Spain.

${ }^{3}$ Instituto Madrileño de Estudios Avanzados en Nanociencia (IMDEA-Nanociencia), C/ Faraday, 9. Cantoblanco 28049, Madrid, Spain.

${ }^{4}$ Department of Physics and Astronomy, Uppsala University, 75237-Uppsala, Sweden

${ }^{5}$ Institute of Physics, Polish Academy of Sciences, 02668-Warsaw, Poland ${ }^{6}$ Departamento Física de la Materia Condensada, Condensed Matter Physics Center (IFIMAC) and Instituto de Física de Materiales "Nicolás Cabrera”, Universidad Autónoma de Madrid, Cantoblanco 28049, Madrid, Spain.

\begin{abstract}
The assembly of two Diphenylethylenediamine enantiomers, separately deposited on $\mathrm{Cu}(100)$, is investigated from the first stages of two-dimensional crystallization to the nucleation and growth of the second layer. By means of Scanning Tunneling Microscopy, we show that the chirality of the enantiomers is expressed at different levels of molecular organization. Below the monolayer completion, a disordered phase coexists with domains of a square lattice aligned with the principal crystallographic directions of the substrate. The intrinsic chirality of the molecules is only manifested through specific features contained within the corresponding unit cell. For increasing coverage, this arrangement is accompanied by a second square structure, which appears to be clockwise or counterclockwise rotated with respect to the $\mathrm{Cu}(100)$ directions depending on the enantiomer. The nucleation of molecular chains on top of the aligned square structure gives rise to a second layer exhibiting a striped-like configuration with remarkable left- or right-handed helicity that mirrors that of the particular enantiomer. The nearly flat configuration of the first layer molecules is confirmed by angular dependent X-ray absorption experiments and supported by molecular dynamics simulations.
\end{abstract}




\section{INTRODUCTION}

Obtaining spatially uniform chiral thin films with atomic-scale control of their handedness is a powerful way for developing nano-devices with novel properties. For example, the relation between magnetism and chirality has lately triggered an emergent field of research on chirality-induced spin selectivity and spin-dependent effects in layers of chiral molecules on surfaces. ${ }^{1,2,3,4,5,6}$ In fact, being chirality a general property, the phenomena associated to it can be found in almost all areas of science and particularly has important implications in biomedical or medicinal applications, where the use of the wrong enantiomer entails high risks. It is therefore stimulating to develop methods that allow distinguishing and separating or selecting the molecules with the desired chiral orientation. This is a difficult task because the energetic differences between enantiomers are expected to be very small and their physico-chemical properties similar. ${ }^{7,8}$ In fact, the main synthetic routes employed nowadays typically rely on the use of chiral catalysts, ${ }^{9}$ frequently of biological origin, ${ }^{10}$ as a means to favor the production of one enantiomer over the other. The efficiency of these agents is based on the effect of chiral recognition, ${ }^{11}$ being biocatalysts highly efficient but delicate, expensive and scarce. ${ }^{12}$ For these reasons a strong interest exists to modify conventional metal-particle catalysts to bestow them with chiral recognition capabilities. The most common approach is to adsorb on the catalysts surface some adequate modifiers creating a chiral environment. ${ }^{13}$ This strategy has prompted a great number of studies focused on elucidating the conditions that lead to the appearance of long-range ordered structures with a well-defined homochiral symmetry suitable for enantioselective binding.

Chirality may manifest on solid surfaces at different levels of molecular assembly, from individual adsorbed species to one-dimensional (1D) molecular chains or twodimensional (2D) molecular clusters and supramolecular patterns. ${ }^{14,15,16,17,18,19,20,21,22}$ Unless important changes occur due to adsorption itself, a chiral molecule is necessarily chiral upon adsorption on a surface and generally assembles forming homochiral ordered 2D structures originated from the asymmetry of the molecule. ${ }^{14}$ The other enantiomer will generate the mirror image systems. ${ }^{23,24}$ Steric effects in lateral interactions between adsorbed molecules are responsible for the segregation of different enantiomers of a racemic mixture into $2 \mathrm{D}$ domains of opposite handedness, ${ }^{25,26}$ but the interaction with the substrate can also provoke deformations of the molecules 
influencing their arrangement on the surface. ${ }^{27}$ Fabricating spatially uniform chiral thin films with atomic-scale control of their handedness is not only relevant for applications; it is also important to reach a profound understanding of the interactions between the chiral building blocks and the mechanisms allowing transmission of their chirality to long-scale arrangements because the details of these structures can have a substantial influence on their enantioselectivity. ${ }^{28}$ In the last decades surface spectroscopies and scanning probe microscopies have provided important insights on chiral 2D assembly which complemented with advances in theoretical modeling have delivered understanding into the balance of interactions that determine the system behavior. ${ }^{26,29,30}$ However, chiral assembling beyond the first layer remains a less investigated topic. ${ }^{31,32}$

With the purpose of exploring the origin of chirality transfer and amplification relevant for the bottom-up processes of crystallization and chiral segregation, we investigate the self-assembly of two enantiomers of the same molecule on a solid, single crystal surface. In particular, the enantiomers of 1,2-Diphenylethylenediamine (DPEDA for short) have been deposited from the vapor phase on $\mathrm{Cu}(100)$ under ultra-high-vacuum (UHV) and room temperature (RT) conditions. DPEDA possesses two chiral centers located at the two carbon atoms of the ethylene chain and presents two enantiomers which are designated according to their handedness at each chiral center and optical activity as $(1 \mathrm{R}, 2 \mathrm{R})-(+)$ and $(1 \mathrm{~S}, 2 \mathrm{~S})-(-)$. Both DPEDA forms adsorb at the $\mathrm{Cu}(100)$ surface at RT displaying diverse ordered structures, as opposed to a similar molecule (1,2-Diphenyl-1,2-ethanediol, DPED) which contains no nitrogen atoms and chemisorbs stably at RT on transition metals such as $\mathrm{Fe}$ or $\mathrm{Co}^{4}$ but adsorbs only weakly at low temperature on $\mathrm{Cu}^{33}$

The supramolecular structures formed by the adsorbed DPEDA layers have been characterized by Scanning Tunneling Microscopy (STM). It is shown that the handedness of the individual DPEDA molecules is transferred at different levels of the molecular organization as a function of increasing coverage. Concomitant Near Edge Xray Absorption Fine Structure (NEXAFS) spectroscopy measurements are consistent with a slightly tilted adsorption geometry of the molecules in the first monolayer. Molecular Dynamics (MD) simulations provide insights on the adsorbate configuration over the substrate where the $\mathrm{N}$ atoms of the molecules (stereogenic centers) are 
coordinated to $\mathrm{Cu}$ surface atoms. This fact produces symmetric footprints for each enantiomer that are highlighted in the molecular assemblies observed experimentally.

\section{METHODS}

STM measurements were carried out at RT in a UHV chamber with a base pressure of $1 \times 10^{-10}$ mbar using a commercial Aarhus SPM 150 with KolibriSensor probes and a Nanonis Control System (SPECS Surface Nano Analysis GmbH). The sharp metallic tip was cleaned in-situ via $\mathrm{Ar}^{+}$sputtering. Topographic STM was conducted in the constant current mode. Typical tunneling parameters were: sample bias voltages of 1-2 V and currents of 100-200 pA. The in-plane lattice constant, in high resolution images, and step heights, in large scale images of an in-situ cleaned $\mathrm{Cu}(111)$ single crystal (see Figure S1 in the Supporting Information) were employed for calibration of the STM piezo scanner .

Substrate cleaning and sample preparation were performed in a dedicated preparation chamber equipped with Low Energy Electron Diffraction (LEED) optics and diverse evaporators connected to the STM characterization chamber. The $\mathrm{Cu}(100)$ single crystal was prepared by repeated cycles of $\mathrm{Ar}^{+}$sputtering $(1 \mathrm{keV})$ plus annealing at $650^{\circ} \mathrm{C}$. After this procedure, the observation by STM of the $\mathrm{Cu}(100)$ surface consisting of large terraces separated by monoatomic steps and the sharpness of the LEED pattern confirmed a well-ordered clean surface that was used to establish the crystal azimuth orientation (see Figure S2 in the Supporting Information) employed throughout the present work as a reference for the different structures observed. Due to the high vapor pressure of 1,2-Diphenylethylenediamine (DPEDA) (Sigma-Aldrich. 97\%), the molecules were deposited from a Knudsen cell without heating, i.e., at RT by simply opening a separation valve, onto the clean substrate kept at RT. The as-prepared samples were directly transferred under UHV for the STM characterization, including local coverage determination from large scale images. Fast Fourier Transform (FFT) analysis of all STM shown was employed to confirm the structural analysis. Due to the complexity of the systems, with coexistence of diverse structures, only surfaces containing one or the other enantiomer were investigated.

NEXAFS measurements were carried out at the HECTOR end-station of the BOREAS beamline of ALBA synchrotron ${ }^{34}$ employing fully linearly polarized light produced by 
an APPLE-II elliptical undulator in the total electron yield (TEY) detection mode. The base pressure during the measurements was better than $1.0 \times 10^{-10}$ mbar. The angular dependent measurements were obtained by rotating the polar angle of the sample and thus the X-ray incidence angle while keeping the polarization in the linear horizontal configuration with the electric field vector of the impinging X-rays lying in the incidence plane (see sketch in Figure 2a). Due to carbon-contamination of the optical elements and to the rather low coverage of DPEDA molecules studied in this work, the C K-edge spectra were obtained by normalizing the signal measured on the DPEDA/Cu(100) sample by the TEY signal measured on a freshly prepared clean substrate. In this way it is possible to almost get rid of the normalization artifacts arising at the carbon K-edge. ${ }^{35}$ All the TEY spectra were first normalized by the impinging photon flux measured as the TEY signal on a freshly evaporated gold mesh placed in between the last optical element and the sample. The enantiopure (R,R) or $(\mathrm{S}, \mathrm{S})$ DPEDA/Cu(100) samples of the NEXAFS experiments were prepared in-situ in the preparation chamber of the HECTOR end-station in a base pressure better than $1.0 \times 10^{-9}$ mbar.

Molecular Dynamics (MD) simulations consist of solving numerically the Newton equations of motion for an atomistic model of the considered system under the appropriate thermodynamic conditions. Here, we performed MD simulations of (S,S)DPEDA molecules adsorbing at a $\mathrm{Cu}(100)$ surface using the NAMD 2.11 software. ${ }^{36} \mathrm{In}$ all our simulations the temperature was maintained constant at $298 \mathrm{~K}$ using a Langevin thermostat. We performed two different simulations. In the first one, we considered the adsorption of a single (S,S)-DPEDA molecule onto a substrate made of $2400 \mathrm{Cu}$ atoms organized in 12 atomic layers with 200 atoms each (area of the surface $\approx 13.11 \mathrm{~nm}^{2}$ ). In a second simulation, 60 (S,S)-DPEDA molecules adsorbed onto a surface consisting of $9600 \mathrm{Cu}$ atoms organized in 12 layers of 800 atoms each (area of the surface $\approx 52.43$ $\mathrm{nm}^{2}$ ), which correspond to a coverage of $1.14 \mathrm{molec} / \mathrm{nm}^{2}$. The DPEDA molecule is modeled using the CHARMM General Force Field for small organic molecules ${ }^{37}$ and the $\mathrm{Cu}$ atoms are modeled with the force field developed by Heinz, ${ }^{38}$ compatible with CHARMM. More methodological details can be found in the Supporting Information. Simulations for the (R,R)-DPEDA are expected to be the same expect for the mirror symmetry. 


\section{RESULTS AND DISCUSSION}

In order to help interpreting the experimental results, we have performed all atomic MD simulations of the DPEDA molecule adsorbed over the $\mathrm{Cu}(100)$ surface at $298 \mathrm{~K}$. In the gas phase, the molecule has a minimum energy configuration that is non planar, as shown in Figure 1a. However, our MD simulations show that at room temperature the molecules adsorb at the substrate with a planar configuration as shown in Figure $1 \mathrm{~b}$ for the $(\mathrm{S}, \mathrm{S})$-DPEDA enantiomer. The adsorbed DPEDA molecules have their $\mathrm{N}$ atoms between $\mathrm{Cu}$ atoms of the top layer in a way that each $\mathrm{N}$ atom coordinates with four $\mathrm{Cu}$ surface atoms. This gives the DPEDA molecule a particular orientation over the surface, as seen in Figure 1c. We estimate a large adsorption free energy of $-75 \mathrm{Kcal} / \mathrm{mol}$ at $298 \mathrm{~K}$ which is due to the large contact surface between molecular rings and the $\mathrm{Cu}$ surface and the high coordination of the molecule $\mathrm{N}$ atoms with $\mathrm{Cu}$ atoms. At this temperature, we predict from the simulations a substantial diffusion of the DPEDA molecule over the substrate. From a simulation of a long trajectory ( $80 \mathrm{~ns}$ ) of a single molecule over the surface, a diffusion coefficient of $1.25 \times 10^{-6} \mathrm{~cm}^{2} / \mathrm{s}$ is estimated. This value for the diffusion coefficient is typical for organic molecules of similar size over metal surfaces. ${ }^{39}$ The motion observed for the molecule is Brownian (see Figure S1 in the Supporting Information), and proceeds via jumps (molecular hopping) between adjacent adsorbing sites which maintain the $\mathrm{N}$ atoms of DPEDA always coordinated with four $\mathrm{Cu}$ atoms. As the molecular density increases, two observable events are worth of mention. Figure 1d depicts a snapshot after a large elapsed time (282 ns) for a MD simulation with a molecular density of $1.14 \mathrm{molec} / \mathrm{nm}^{2}$ at $298 \mathrm{~K}$. Magnifications corresponding to the regions marked in Figure $1 \mathrm{~d}$ by blue and red shallow color are shown in Figures 1e and 1f, respectively. On the one hand, as indicated by the orange rectangles in Figure 1e, an incipient alignment of the molecules (molecular chains) is observed that, taking into account the handedness of the particular enantiomer, anticipates the occurrence of mirror-like striped phases for the opposite isomers that are indeed found for high coverages (see below). On the other hand, the phenyl rings of some molecules slightly lift out from the surface plane (red arrows in Figure 1f) and due to lateral interactions between molecules, eventually all molecules would adopt a similarly discretely tilted configuration. This hypothesis is supported by NEXAFS measurements performed to provide insights about the orientation of the molecules in the first layer. 


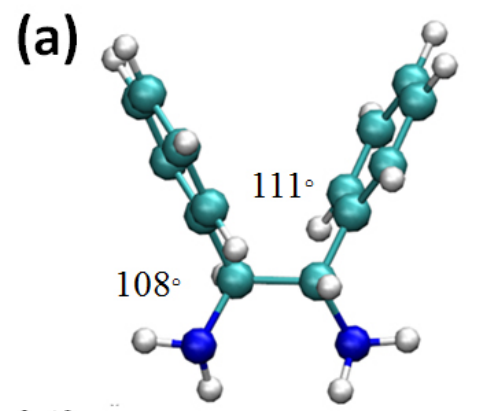

(d)

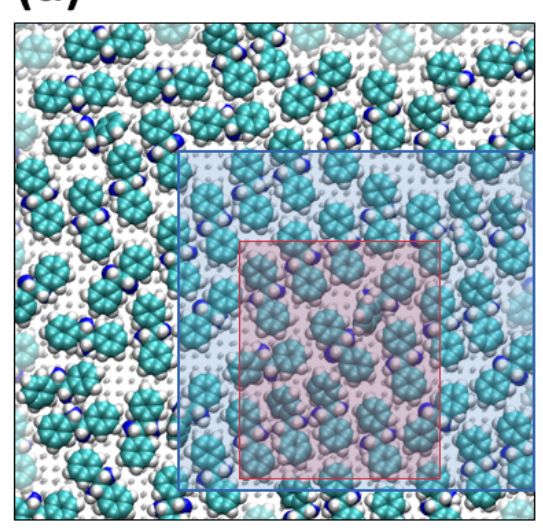

(b)

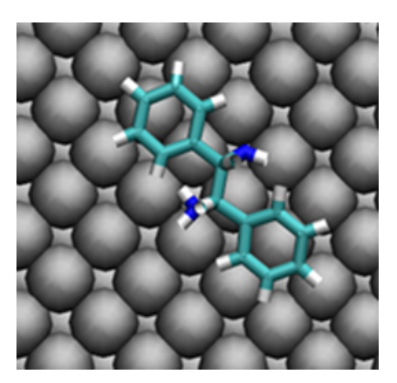

(e)

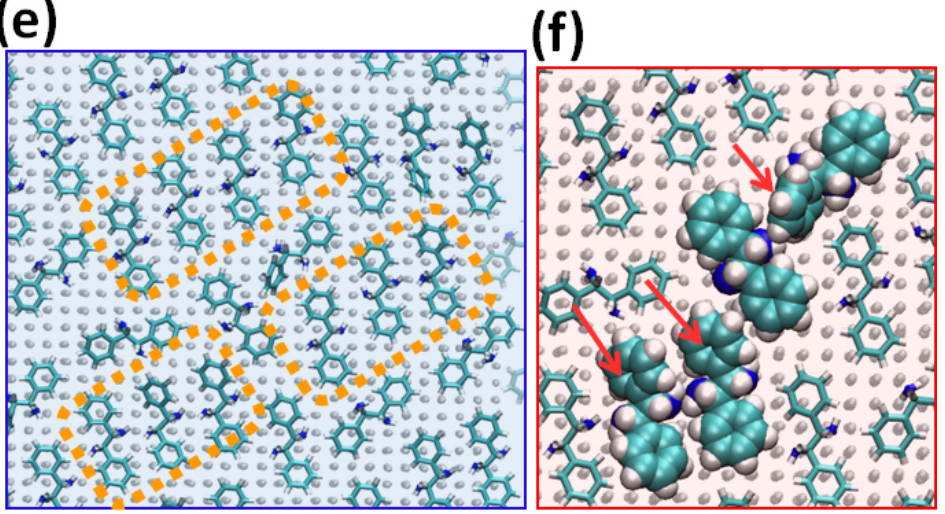

Figure 1. (a) Minimum energy configuration for the (S,S)-DPEDA molecule (CPK representation) (b) Snapshot (top view) from a MD simulation at $298 \mathrm{~K}$ of the (S,S)DPEDA molecule adsorbed over the $\mathrm{Cu}(100)$ surface. The $\mathrm{Cu}$ atoms are shown as van der Waals spheres and the DPEDA molecule is shown in bond representation (c) lateral view of (b) with the DPEDA molecule shown either in van der Waals representation to emphasize the contact between the surface and the molecule (top) or bonds representation to highlight the location of nitrogen atoms (bottom). (d) Snapshot after a large elapsed time (282 ns) for a MD simulation with a molecular density of 1.14 molec $/ \mathrm{nm}^{2}$ at $298 \mathrm{~K}$. (e-f) Different size magnifications corresponding to the regions marked in (d) by blue and red shallow color, respectively. Parallel molecules forming rows are enclosed in orange rectangles and some lifted phenyl rings by red arrows. The employed colors for each atom are $\mathrm{N}$ : blue, $\mathrm{C}$ : cyan, $\mathrm{H}$ : white, $\mathrm{Cu}$ : grey. Images made with Visual Molecular Dynamics program. ${ }^{40}$

For each DPEDA enantiomer, the amine groups are equally oriented with respect to the axis connecting the two phenyl rings but oppositely oriented for one enantiomer with respect to the other. As expected and supported by the above MD simulations, upon 
adsorption on the metal surface the two amine groups will orient towards the copper surface plane and, as a consequence, adsorbed (R,R)-DPEDA and (S,S)-DPEDA can be seen as mirror-like S-shaped units or symmetric footprints as depicted in Figures 2a and $2 \mathrm{~b}$, respectively. Figure 2 shows the incident angle dependent linearly polarized C Kedge data for (R,R)-DPEDA and (S,S)-DPEDA on $\mathrm{Cu}(100)$ respectively for an estimated coverage of about one monolayer of molecules. The $\mathrm{C} \mathrm{K}$-edge presents a strong white line in the region corresponding to the $\pi *$ states $(\approx 283-286 \mathrm{eV})$ and is less featured at higher photon energies in correspondence with $\sigma^{*}$ transitions. It also shows a large linear dichroism at the $\pi^{*}$ states indicating that the molecules adsorb in a slightly tilted configuration on the $\mathrm{Cu}(100)$ surface. Though the intensity is smaller at the N-K edge, due to the lower number of $\mathrm{N}$ atoms per molecule, a strong linear dichroism in the corresponding $\pi^{*}$ region $(\approx 398-400 \mathrm{eV})$ is also observed (Figure S4 in the Supporting Information).
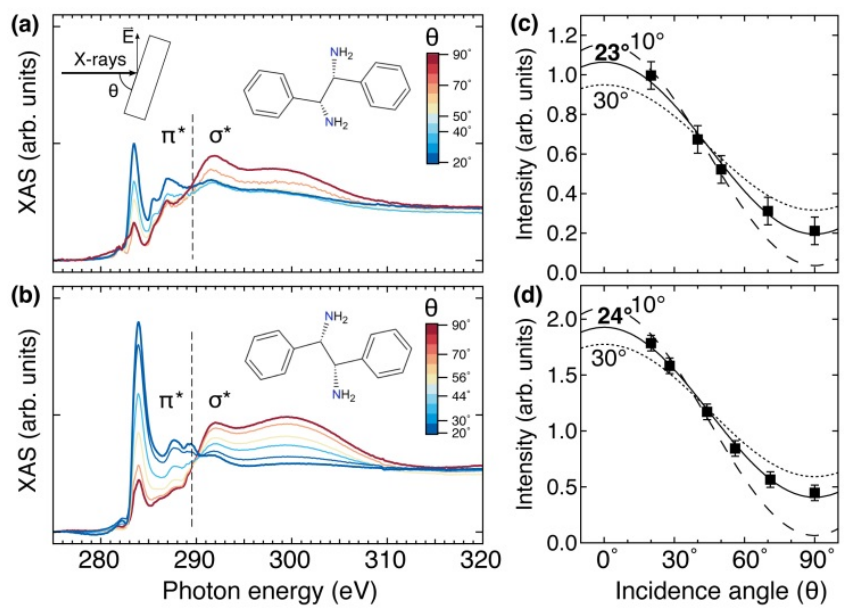

Figure 2. Angular dependence of the NEXAFS intensity for the $\mathrm{C} \mathrm{K}$-edge in the case of (R,R)-DPEDA (a) and (S,S)-DPEDA (b) on $\mathrm{Cu}(100)$. The intensity of the corresponding $\pi^{*}$ peak is plotted in (c) and (d), respectively, along with the expected evolution for diverse molecular tilts. The chemical structure of the enantiomers is inserted in the corresponding panels with the $\mathrm{N}$ atoms depicted inwards so that they can be seen as mirror-like S-shaped units.

More quantitative insights on the adsorption geometry can be obtained by modeling the angular dependent intensity variation of the carbon $\pi^{*}$ resonance. In particular, the intensity variation for a $\pi$ orbital adsorbed on a fourfold symmetry substrate has a 
dependence on the linearly polarized X-ray incidence angle $\theta$ given by $I=$ $I_{0} \cos ^{2}(\theta-\alpha)$, where $\alpha$ is the average tilt angle of the $\pi$ orbital with respect to the surface normal. Figures $2 \mathrm{c}$ and $2 \mathrm{~d}$ show the least-squares fits to the intensity of the $\pi^{*}$ peak as a function of the X-ray incident angle with the former expression, that results in a best fitting average tilt angle of $\approx 24^{\circ} \pm 5^{\circ}$ for the $(\mathrm{S}, \mathrm{S})$ and $23^{\circ} \pm 5^{\circ}$ for the $(\mathrm{R}, \mathrm{R})$ enantiomer. It is worth noting that the average tilt angle obtained here does not take into account any possible angle between the two phenyl rings of the DPEDA molecules, being only an estimate of the average angle of the pi orbitals with respect to the sample surface. In the same figure the intensity curves for average tilt angles of $30^{\circ}$ and $10^{\circ}$ are also plotted in order to give an estimate of the fit significance. In agreement with the MD simulations for large molecular densities, the NEXAFS results are consistent with a mostly planar adsorption configuration of the $(\mathrm{R}, \mathrm{R})$ - and $(\mathrm{S}, \mathrm{S})-\mathrm{DPEDA}$ molecules with the two phenyl rings slightly lifted from the $\mathrm{Cu}(100)$ surface.

Before presenting and discussing the STM results, it is worth mentioning that due to adsorption/desorption of molecules onto/from the tip during scanning, imaging conditions were observed to change with the consequent variations in resolution and molecular-scale details even within the same image. For this reason, we have concentrated on the analysis of the long-range ordered structures rather than on the fine details regarding the inner structure of the unit cells. Unless commented, all conclusions in the following are supported by accurate determination of the unit cell dimensions and periodicities of the different structures based on statistical analysis of a dataset of nearly one hundred STM images and the FFT analysis of a selection of the most representative. The employed approach, including crystallographic references, permits the unambiguous ascription of the surface structures in terms of in-plane lattice parameters and orientation. Though in some cases the features observed in the STM images allow establishing a relation with the chirality of the molecules, assessing the exact unit cell molecular content and arrangement would imply a comparison with a theoretical simulation of the topographic STM images that has not been carried out.

Chiral asymmetry can be transferred from the single molecule to the mesoscale by means of non-covalent assemblies and direct observation by STM has demonstrated that, commonly, molecular conformation adjustment leads to the formation of chiral arrangements. $^{23}$ This recognition process between adjacent molecules permits the 
occurrence of enantiopure phases on surfaces. However, for adsorbed layers on a solid surface the resulting structure(s) comes from the interplay between the moleculemolecule and molecule-substrate interactions. Thus, different molecular structures and bonding motifs leading to extended chiral networks can be realized. In the following diverse modes of chiral organization will be described for increasing coverage for surfaces containing one DPEDA enantiomer. The structures detailed below (or their mirror image) were found for both enantiomers and, therefore, in some cases only one of them is used to illustrate the findings.
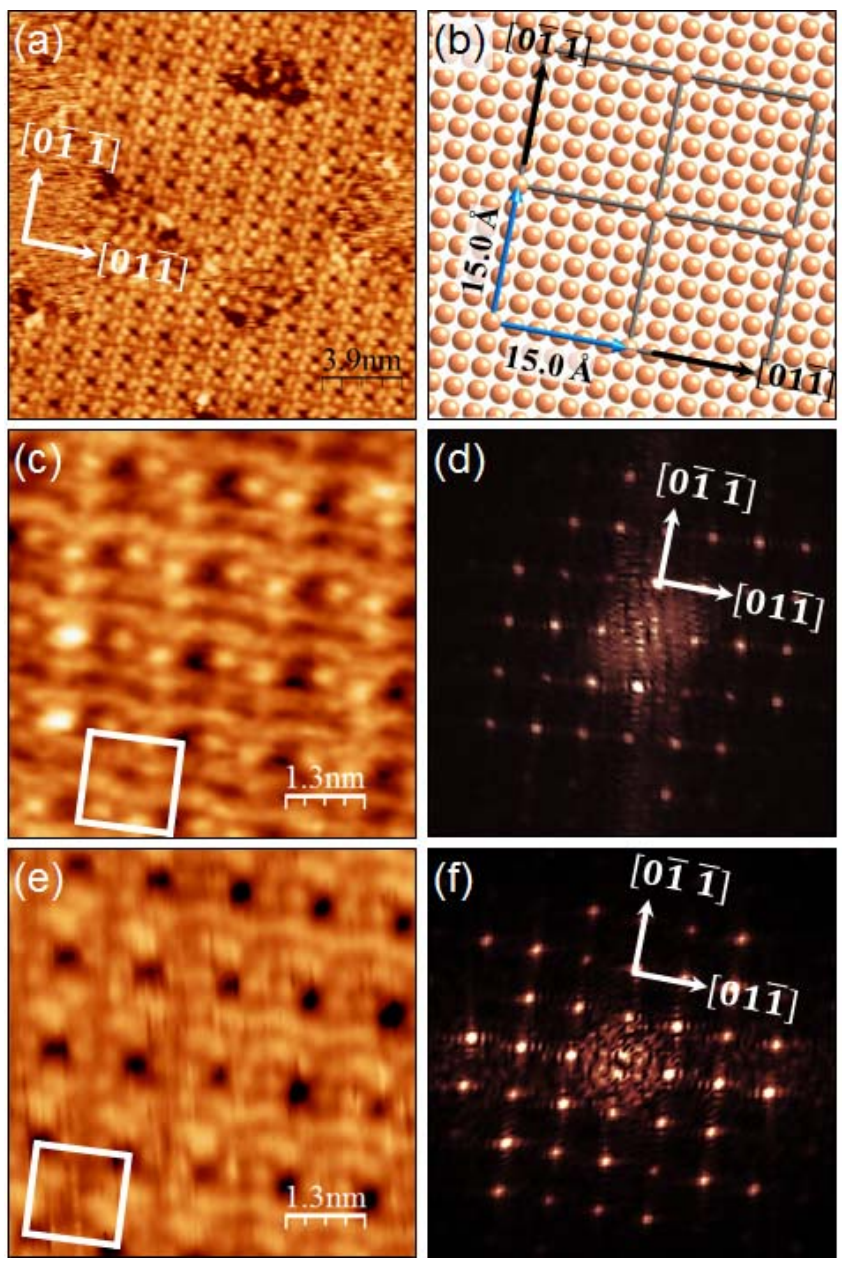

Figure 3. (a) Topographic STM of a surface region of (R,R)-DPEDA, deposited on $\mathrm{Cu}(100)$ at $\mathrm{RT}$, containing the aligned square lattice (A-SQ). Image size: $19.5 \mathrm{~nm} \times 19.5$ $\mathrm{nm}$. (b) Unit cell of a (6x6) commensurate lattice, the orange balls indicate the copper atoms of the substrate. (c),(e) and (d),(f) are STM images and FFTs for (S,S)-DPEDA and $(\mathrm{R}, \mathrm{R})-\mathrm{DPEDA}$, respectively. The crystallographic orientation of the substrate is common for all panels. STM parameters: (a) $\mathrm{I}=125 \mathrm{pA}$, bias $=+1.2 \mathrm{~V}$, (c) $\mathrm{I}=200 \mathrm{pA}$, bias $=+1.5 \mathrm{~V}$ and $(\mathrm{e}) \mathrm{I}=200 \mathrm{pA}$, bias $=+1.7 \mathrm{~V}$. 
For low deposition times, no ordered structures of DPEDA/Cu(100) were observed at RT. However, close to monolayer (ML) completion disordered areas were found to coexist with long-range ordered regions with lateral sizes of several tens of nanometers. These domains exhibit a chessboard appearance (Figure 3a) where the molecules arrange forming a $2 \mathrm{D}$ lattice aligned with the [0 $\overline{1} \overline{1}]$ and [01 $\overline{1}$ ] crystallographic directions of $\mathrm{Cu}(100)$ as indicated in the figure. The dimensions of this square lattice $(a$ $=b \approx 1.5 \mathrm{~nm} \pm 0.1 \mathrm{~nm}$ ), referred to as A-SQ (aligned square) from now on, are in accordance with a $(6 \times 6)$, or $(60,06)$ in matrix notation, commensurate lattice $(1.53 \mathrm{~nm}$ $\times 1.53 \mathrm{~nm})$ with respect to the underlying substrate $\left(a_{100}=2.55 \AA\right)$ and depicted in Figure $3 b$. The same structure is found for both enantiomers, (S,S)-DPEDA (Figures 3c3d) and (R,R)-DPEDA (Figures 3e-3f). Because the unit cell is parallel to the main crystallographic directions, no difference exists in the lattice orientation between samples prepared from each enantiomer. The intrinsic chirality of the molecules, and the preservation of their chiral centers upon adsorption, is only manifested within the unit cell (u. c.) thanks to submolecular details reflecting the sense of handedness of each building block (see also Figure S5 in the Supporting Information). However, though up to 9-10 protrusions per u.c. can be identified in some high resolution images (see Figure S6 in the Supporting Information), neither single molecule adscription nor exact molecular density (number of molecules per u. c.) can be inferred.

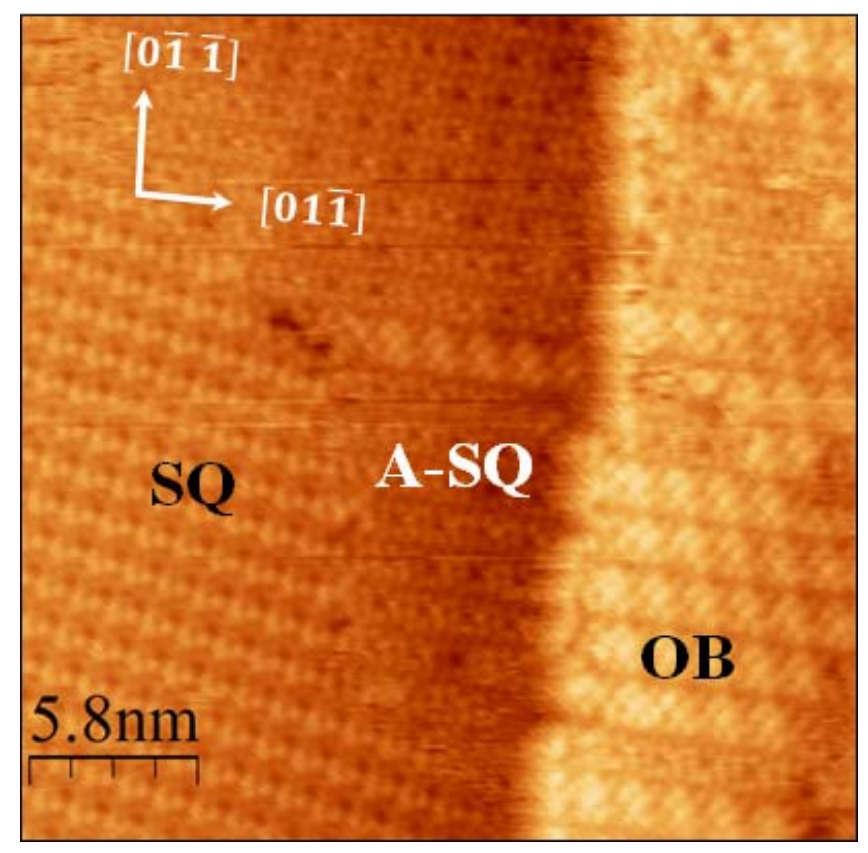


Figure 4. Topographic image of (S,S)-DPEDA covering two adjacent terraces separated by one monoatomic step of the $\mathrm{Cu}(100)$ substrate. Three different structures labeled as A-SQ (aligned square), SQ (square) and OB (oblique) are detailed in the text. STM parameters: $\mathrm{I}=200 \mathrm{pA}$, bias $=+2.0 \mathrm{~V}$.

With increased exposure, the metallic substrate is fully covered. Figure 4 shows a surface region containing two terraces, separated by one monoatomic step of the underlying $\mathrm{Cu}(100)$. Coexistence of different molecular structures permits unambiguously determining the relationships between them. At the lower terrace, in addition to the above described A-SQ, a structure with a smaller square lattice (denoted as SQ) is observed and it is rotated with respect to the reference [011] direction. Long range ordered domains seem to be formed by incorporation of molecules at the disordered regions observed at low coverages (Figure 3a). Finally, at the upper terrace, a third molecular arrangement (labeled OB in the image) is observed. It corresponds to the nucleation of a second layer and will be described later on. 

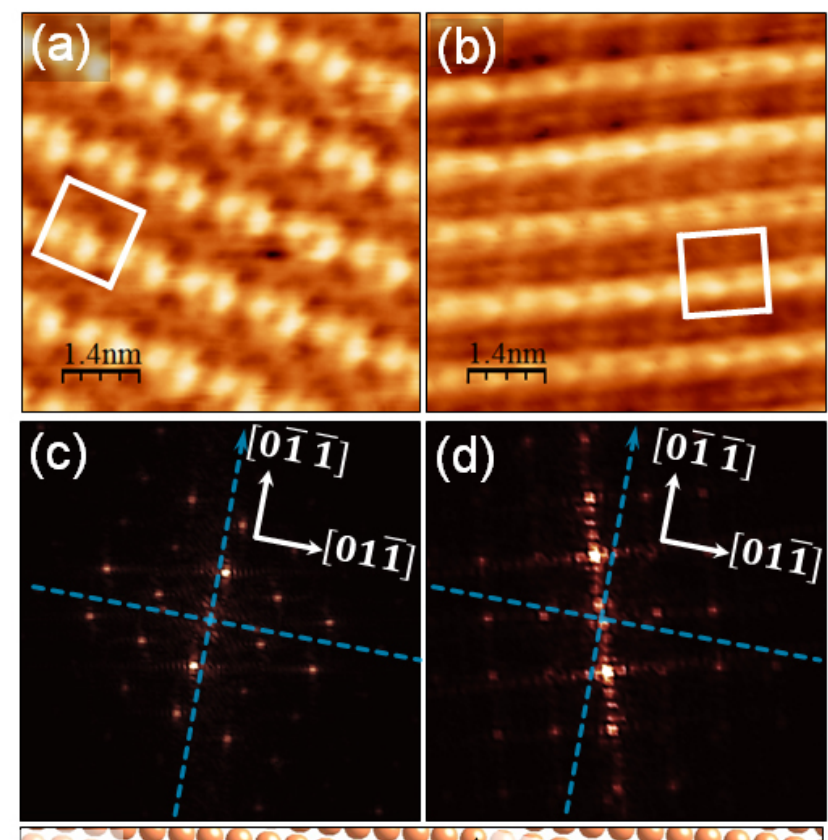

(e)

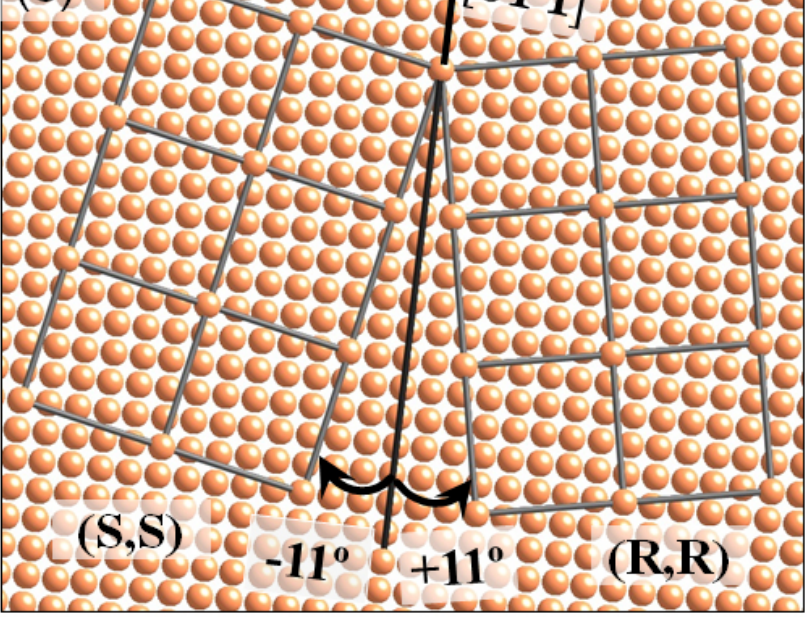

Figure 5. Topographic images and their corresponding FFT patterns for the SQ structure of $(\mathrm{S}, \mathrm{S})$-DPEDA $(\mathrm{a}, \mathrm{c})$ and $(\mathrm{R}, \mathrm{R})$-DPEDA $(\mathrm{b}, \mathrm{d})$ on $\mathrm{Cu}(100)$. The orientation of

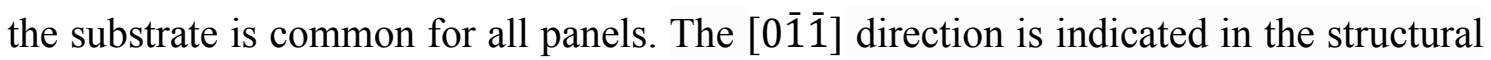
model in (e) schematizing the mirror-like domains rotated by $-11^{\circ}$ and $+11^{\circ}$ observed in (a) and (c) for (S,S) and (R,R), respectively. STM parameters: (a) I = 200 pA, bias = $+2.0 \mathrm{~V}$ and (b) $\mathrm{I}=105 \mathrm{pA}$, bias $=-1.1 \mathrm{~V}$.

Topographic images of the SQ structure and the corresponding FFTs are shown in Figures $5 \mathrm{a}-5 \mathrm{c}$ and $5 \mathrm{~b}-5 \mathrm{~d}$ for $(\mathrm{S}, \mathrm{S})-\mathrm{DPEDA}$ and $(\mathrm{R}, \mathrm{R})-\mathrm{DPEDA}$, respectively. The rotation by $\pm 11^{\circ}$ of the lattice with respect to the substrate crystallographic directions breaks the mirror symmetry. Conversely to what happens for the A-SQ, where no differences between the lattices for the two stereoisomers exist, as the density of the 
molecular layer increases and the SQ structure appears, the lateral interactions between the chiral molecular units start to dominate and the handedness of the single enantiomer is here expressed into the lattice handedness. Thus, images obtained for one or the other enantiomer are mirror-like (Figures 5a-5b) with respect to the plane perpendicular to the

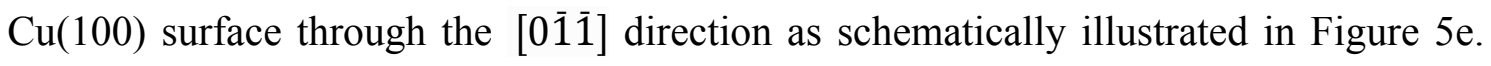
As expected from the square substrate symmetry, domains rotated $90^{\circ}$ were found on different surface terraces (see Figure S7 in the Supporting Information). Larger areas of the SQ structure for each enantiomer are shown in Figure 4 (left) and Figure S8. The unit cell dimensions $\left(a_{S Q}=b_{S Q} \approx 1.3 \mathrm{~nm} \pm 0.1 \mathrm{~nm}\right)$ and rotation angle $\left(\approx \pm 11^{\circ}\right)$ are in agreement with a commensurate structure with respect to the $\mathrm{Cu}(100)$ surface lattice that can be specified in matrix notation as $(5-1,15)$ and $(51,-15)$ for (S,S)-DPEDA and $(\mathrm{R}, \mathrm{R})-\mathrm{DPEDA}$, respectively. These results show that chirality is expressed at two length scales with increasing coverage during the 2D crystallization of both enantiomers of DPEDA: a basic level (point chirality) corresponding to the chiral character of adsorbed molecules that is present in both, A-SQ and SQ lattices, and a second level (organizational chirality) that emerges as a mirror assembly in the SQ lattice.

As commented above and observed in Figure 4, nucleation and growth of a second layer takes place in some regions. Figure 6 shows an image of the $\mathrm{Cu}(100)$ surface covered by $(\mathrm{R}, \mathrm{R})$-DPEDA above the monolayer. As it can be seen in Figure 6a, on top of the ASQ lattice some molecules group forming linear chains (marked by an arrow) that run parallel to the [011] direction. Eventually, these molecular chains coalesce laterally forming striped domains of a second layer as seen at the lower part of the image. The observed structure can be described by an oblique (OB) unit cell, with dimensions $a_{O В} \approx$ $1.5 \mathrm{~nm} \pm 0.1 \mathrm{~nm}$ and $c_{О В} \approx 1.8 \mathrm{~nm} \pm 0.1 \mathrm{~nm}$ which correspond to a $(60,36)$ lattice commensurate with the substrate. Taking advantage of the coexistence of both lattices in the same surface region, some additional structural details including the relationship between the OB and the A-SQ can be extracted. Figures $6 \mathrm{~b}$ and $6 \mathrm{c}$ are the FFT of the areas marked with light blue and green lines in Figure 6a and are used to separately determine the relative orientation and size of the two structures. The unit cells of the two lattices are depicted in Figure $6 \mathrm{~d}$ and their relationship in matrix notation is $(10$, $1 / 21)$. 

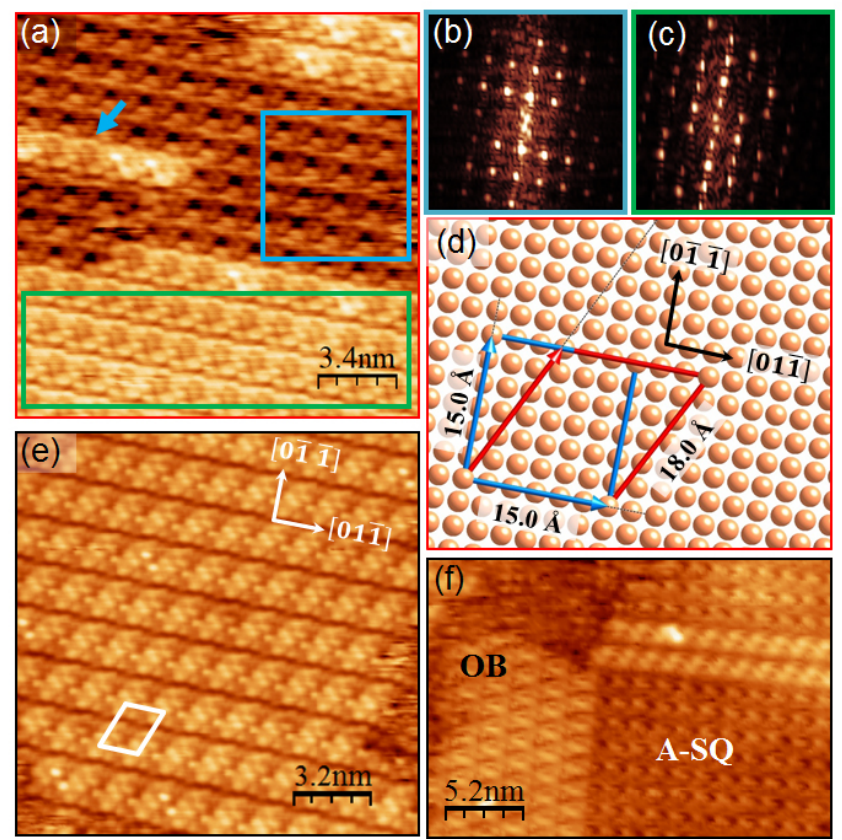

Figure 6. (a) Topographic STM image for a local coverage of $\approx 1.2 \mathrm{ML}$ of $(\mathrm{R}, \mathrm{R})-(+)$ DPEDA. The chain (blue arrow) is aligned with the [011] direction. (b) and (c) display the 2D FFT patterns of the A-SQ and OB regions, marked in (a) by a blue square and a green rectangle, respectively. (d) Unit cells for both lattices. STM images showing: (e) a $2 \mathrm{ML}$ region and (f) an area with two perpendicular domains of the $\mathrm{OB}$ on top of the same A-SQ region. The crystallographic orientation of the substrate indicated in (d) and (e) is common for all panels. STM parameters: (a) $\mathrm{I}=105 \mathrm{pA}$, bias $=+1.1 \mathrm{~V}$, (e) $\mathrm{I}=$ $200 \mathrm{pA}$, bias $=+1.0 \mathrm{~V}$, and (f) $\mathrm{I}=133 \mathrm{pA}$, bias $=-0.71 \mathrm{~V}$.

It is worth emphasizing that rather than a compact 2D packing, the second layer assembly shows a quite substantial inter-chain separation but a dense internal structure along the chains. This circumstance reflects a unidirectional "lock-and-key" assembling process $^{41}$ driven by a stronger intra-chain than inter-chain interaction. The OB structure overspreads the whole surface and either large single domains (Figure 6e) or neighboring $90^{\circ}$ rotated domains (Figure 6f) can be found. We note that the nucleation and growth of the second layer occurs selectively, i.e., only on the A-SQ regions and not on top of the SQ as it can be observed in Figure 4 and Figure 6 . The different appearance of the second layer in the shown STM images arises from the change in imaging resolution due to different tip conditions. However, subunit details clearly visible in all cases indicate a supramolecular organization in the form of homochiral 
chains. It is remarkable that all images point out a spiral structure that can be visualized as consisting of left-handed helical chains in the case of (R,R)-DPEDA (Figure 6e) and right-handed in the case of (S,S)-DPEDA (Figure 7a). We suggest that the chiral conformation at the molecular level in the A-SQ domains (Figure 3) favors chiral recognition governed by intermolecular interactions that amplify and transfer chirality. Additional STM data for both enantiomers can be found in Figure S9 of the Supporting Information.
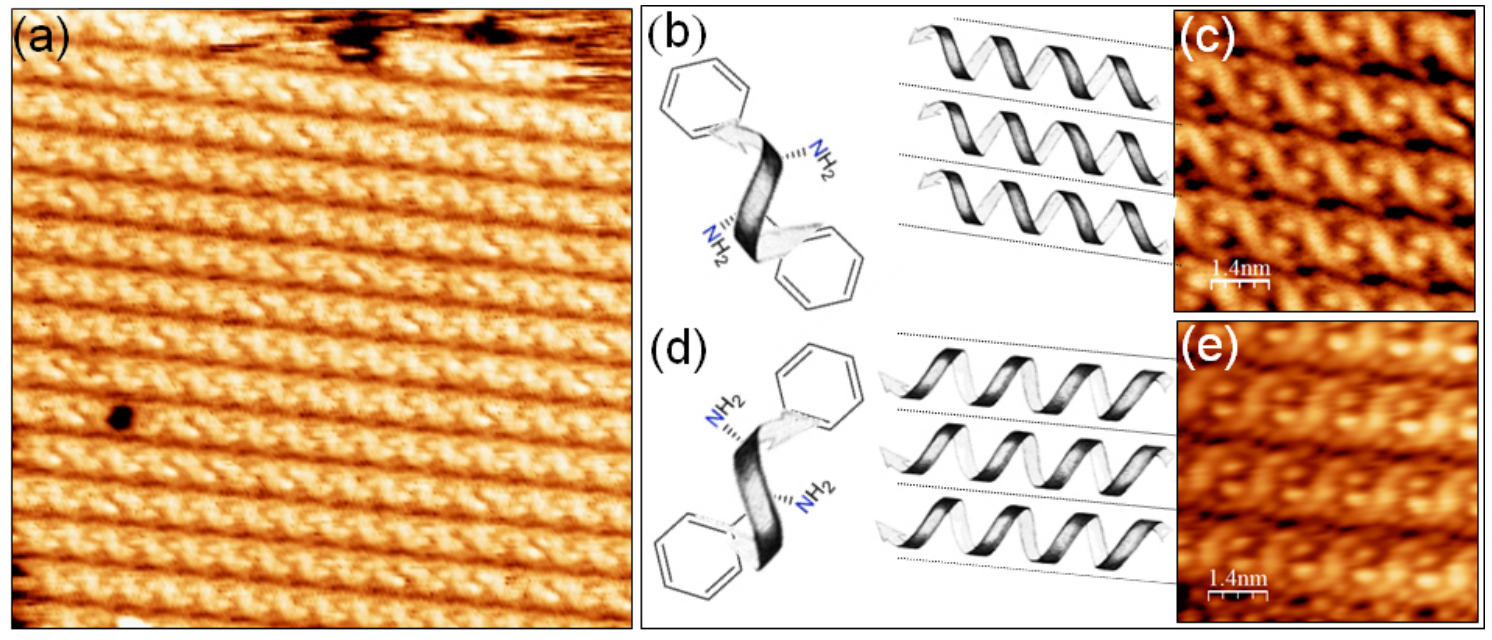

Figure 7. (a) Topographic STM image $(24 \mathrm{~nm} \times 24 \mathrm{~nm})$ of the OB structure of $(\mathrm{S}, \mathrm{S})$ DPEDA. $(b, d)$ Schematic models indicating the enantiomers and structure handedness and (c, e) high resolution images $(7 \mathrm{~nm} \times 7 \mathrm{~nm})$ for (S,S)-DPEDA and (R,R)-DPEDA, respectively. The crystallographic orientation of the substrate is the same as in Figure 6 and the same for all panels. STM parameters: (a) $\mathrm{I}=200 \mathrm{pA}$, bias $=+2.0 \mathrm{~V}$, (c) $\mathrm{I}=199$ $\mathrm{pA}$, bias $=+2.0 \mathrm{~V}$, and (e) $\mathrm{I}=300 \mathrm{pA}$, bias $=-1.0 \mathrm{~V}$.

As brought to mind from the MD simulations, the chirality of the growing organic film is clearly revealed by comparing the molecular scale details observed in the high resolution images of Figure $7 \mathrm{c}$ and $7 \mathrm{e}$, which correspond to (S,S)-DPEDA and (R,R)DPEDA, respectively. The string-like patterns observed for each enantiomer show an evident clockwise and counterclockwise helicity aligned to the [011] direction where all fine details are nicely reproduced. The threaded shape of the structure for each DPEDA enantiomer is mimicked by the respective cartoons in the central panels (Figures $7 \mathrm{~b}$ and 7d). 


\section{CONCLUSIONS}

The results presented here demonstrate that during the initial stages of the crystallization of the single enantiomers (R,R)-DPEDA and (S,S)-DPEDA, the chirality of these molecules is expressed at different length scales depending on the molecular density. Below the completion of the first molecular layer, a first ordered structure labeled A-SQ is observed for both enantiomers with a unit cell aligned with the $\mathrm{Cu}(100)$ surface. This structure, which is obviously non-chiral, is dictated by the interaction between the $\mathrm{N}$ atoms in the DPEDA molecules and the $\mathrm{Cu}$ substrate. At this point, the only information about the chirality in the system is contained within the unit cell and associated to the individual molecules. As deposition proceeds and more DPEDA molecules are accommodated within the first layer, this A-SQ structure coexists with domains of a second denser phase with square unit cell (SQ) rotated by $\pm 11^{\circ}$ with respect to the principal directions of the $\mathrm{Cu}(100)$ substrate for the $(\mathrm{R}, \mathrm{R})$ and $(\mathrm{S}, \mathrm{S})$ enantiomers, respectively. This observation reveals that, as the molecules become closer together, their lateral interactions start to counteract the effect of the substrate. Thus, the chirality of the single enantiomers is amplified and expressed in the clockwise or counterclockwise orientation of the 2D lattice. Finally, as the second layer starts to populate, a third, striped-like structure with oblique (OB) unit cell appears. At this stage the influence of the $\mathrm{Cu}$ substrate is practically negligible and the molecular assembly is driven by the interaction between molecules, with steric effects giving rise to a lockand-key effect leading to the formation of left- or right-handed helicity chains that mirror the handedness of their constituting enantiomers. These findings highlight the fundamental role played by the balance of interactions that govern the transfer of chirality from the point (single molecule level) to the formation of extended assemblies with well-defined handedness, which is the basis of chiral recognition and 3D supramolecular organization.

\section{SUPPORTING INFORMATION}

Methodological details as well as additional STM and NEXAFS experimental data are provided. 


\section{AUTHOR INFORMATION}

\section{Corresponding author}

*E. Barrena (ebarrena@icmab.es)

*C. Ocal (cocal@icmab.es)

\section{ACKNOWLEDGMENT}

This work has been supported by the Spanish Government under projects MAT201347869-C4, MAT2014-59315-R, MAT2015-72848-EXP /AEI, FIS2016-74893-P and MAT2016-77852-C2-1-R (AEI/FEDER, UE) and the Generalitat de Catalunya 2017 SGR 668. We also appreciate the specific agreement between ICMAB-CSIC and the Synchrotron Light Facility ALBA and the Spanish MINECO through the project MAT2015-68994-REDC and the 'Severo Ochoa' Program for Centers of Excellence in R\&D (SEV-2015-0496). The NEXAFS experiments were performed at BOREAS beamline at ALBA Synchrotron with the collaboration of ALBA staff. IMDEA Nanociencia acknowledges support from the 'Severo Ochoa' Program for Centres of Excellence in R\&D (MINECO, Grant SEV-2016-0686). R. P.-R. thanks financial support through BES-2014-067942 (FPI fellowship). We acknowledge the CESGA Supercomputing Center for computational time at the Finisterrae Supercomputer and technical assistance. 


\section{REFERENCES}

(1) Naaman, R.; Waldeck, D. H. Spintronics and chirality: spin selectivity in electron transport through chiral molecules. Annu. Rev. Phys. Chem. 2015, 66, 263-81.

(2) Banerjee-Ghosh, K. et al., Separation of enantiomers by their enantiospecific interaction with achiral magnetic substrates. Science 2018, 360,1331-1334.

(3) Michaeli, K.; Kantor-Uriel, N.; Naaman, R.; Waldeck, D.H. The electron's spin and molecular chirality - how are they related and how do they affect life processes?. Chem. Soc. Rev. 2016, 45, 6478-6487.

(4) Niño, M. Á.; Kowalik, I. A.; Luque, F. J. ; Arvanitis, D.; Miranda, R.; de Miguel, J. J. Enantiospecific Spin Polarization of Electrons Photoemitted Through Layers of Homochiral Organic Molecules. Adv. Mater. 2014, 26, 7474-7479.

(5) Göhler, B.; Hamelbeck, V.; Markus, T. Z.; Kettner, M.; Hanne, G. F. ; Vager, Z.; Naaman, R.; Zacharias, H. Spin Selectivity in Electron Transmission Through SelfAssembled Monolayers of Double-Stranded DNA. Science 2011, 331, 894-897.

(6) Mathew, S.P.; Mondal, P.Ch.; Moshe, H.; Mastai, Y.; Naaman, R. Non-magnetic organic/inorganic spin injector at room temperature. App. Phys. Lett. 2014, 105, 242408.

(7) Mason S. F.; Tranter, G. E. The electroweak origin of biomolecular handedness, Proc. R. Soc. Lond. A, 1985, 397, 45-65.

(8) Quack. M. How important is parity violation for molecular and biomolecular chirality. Angew. Chem. Int. Ed. 2002, 41, 4618-4630.

(9) Ojima I. (Ed.), “Catalytic Asymmetric Synthesis” (VCH, Weinheim, 1993).

(10) Poppe, L.; Novak, L. Selective Biocatalysis. (VCH, Weinheim, 1992).

(11) Zehnacker, A.; Suhm, M. Chirality recognition between neutral molecules in the gas phase. Angew. Chem. Int. Ed. 2008, 47, 6970-6992.

(12) Desantis, G. Enzymes in Organic Synthesis. Modern Drug Discovery, 2002, $43-47$.

(13) Baddeley, C. J.; Jones, T. E.; Trant, A. G.; Wilson, K. E. Fundamental Investigations of Enantioselective Heterogeneous Catalysis. Top. Catal. 2011, 54, $1348-1356$.

(14) Barlow, S. M.; Raval, R. Complex organic molecules at metal surfaces: bonding, organisation and chirality. Surf. Sci. Rep. 2003, 50, 201-341. 
(15) Raval, R. Chiral expressions at metal surfaces. Curr. Opin. Solid State Mater. Sci. 2003, 7, 67-74.

(16) Messina, P.; Dmitriev, A.; Lin, N.; Spillmann, H.; Abel, M.; Barth, J. V.; Kern, K. Direct Observation of Chiral Metal-Organic Complexes Assembled on a $\mathrm{Cu}(100)$ Surface. J. Am. Chem. Soc. 2002, 124, 14000-1.

(17) Chen, T.; Wang, D.; Wan, L.-J. Two-dimensional chiral molecular assembly on solid surfaces: formation and regulation. Nat. Sci. Rev. 2015, 2, 205-216.

(18) Gomar-Nadal, E.; Puigmartí-Luis, J.; Amabilino, D.A. Assembly of functional molecular nanostructures on surfaces. Chem. Soc. Rev. 2008, 37, 490-504.

(20) Humblot, V.; Ortega Lorenzo, M.; Baddeley, Ch.J.; Haq, S.; Raval, R. Local and Global Chirality at Surfaces: Succinic Acid versus Tartaric Acid on Cu(110). J. Am. Chem. Soc. 2004, 126, 6460-6469.

(21) Totani, R.; Methivier, Ch.; Cruguel, H.; Pradier, C.M. ; Humblot. V. Deciphering the Adsorption Mechanisms of RGD Subunits: L-Aspartic Acid on Cu(110). J. Phys. Chem. C 2017, 121, 15842-15850.

(22) Dutta, S.; Gellman,A.J. Enantiomer surface chemistry: conglomerate versus racemate formation on surfaces. Chem. Soc. Rev., 2017, 46, 7787-7839.

(23) Ernst, K.-H. Molecular chirality in surface science. Surf. Sci. 2013, 613, 1-5.

(24) Ernst, K.-H. Molecular chirality at surfaces. Phys. Stat. Solid. B 2012, 249, $2057-$ 2088.

(25) Yablon, D. G.; Giancarlo, L. C.; Flynn, G. W. Manipulating Self-Assembly with Achiral Molecules: An STM Study of Chiral Segregation by Achiral Adsorbates. J. Phys. Chem. B 2000, 104, 7627-7635.

(26) Pérez-García, L.; Amabilino, D.B. Spontaneous resolution, whence and whither: from enantiomorphic solids to chiral liquid crystals, monolayers and macro- and supramolecular polymers and assemblies. Chem. Soc. Rev. 2007, 36, 941-967.

(27) Darling, G. R.; Forster, M.; Lin, C.; Liu, N.; Raval, R.; Hodgson, A. Chiral segregation driven by a dynamical response of the adsorption footprint to the local adsorption environment: bitartrate on $\mathrm{Cu}(110)$. Phys. Chem. Chem. Phys. 2017, 19, $7617-7623$.

(28) Lorenzo, M. O.; Baddeley, C. J.; Muryn, C.; Raval, R. Extended surface chirality from supramolecular assemblies of adsorbed chiral molecules. Nature 2000, 404, 376379

(29) Zaera, F. Chirality in adsorption on solid surfaces. Chem. Soc. Rev. 2017, 46, 7374-7398. 
(30) Latter, E. G.; Raval, R. John Wiley \& Sons, Ltd, 2016, Ch. 10, 285-306.

(31) Mairena, A.; Zoppi, L.; Seibel, J.; Tröster, A.F.; Grenader, K.; Parschau, M.; Terfort, A.; Ernst.K.-H. Heterochiral to Homochiral Transition in Pentahelicene 2D Crystallization Induced by Second-Layer Nucleation. ACS Nano 2017, 11, 865-871.

(32) Parschau, M.; Ernst, K.-H. Disappearing Enantiomorphs: Single Handedness in Racemate Crystals. Angew. Chem. Int. Ed. 2015, 54, 14422 -14426.

(33) Luque, F.J.; Niño, M. Á.; Spilsbury, M. J.; Kowalik, I. A.; Arvanitis, D.; de Miguel, J.J. Enantiosensitive Bonding of Chiral Molecules on a Magnetic Substrate Investigated by Means of Electron Spectroscopies, Chimia, 2018, 72, 418-423.

(34) Barla, A.; Nicolás, J.; Cocco, D.; Valvidares, S. M.; Herrero-Martín, J.; Gargiani, P.; Moldes, J.; Ruget, C.; Pellegrin, E.; Ferrer, S. Design and performance of BOREAS, the beamline for resonant X-ray absorption and scattering experiments at the ALBA synchrotron light source. J. Synchrotron Rad., 2016, 23, 1507-1517.

(35) Stohr, J.; Outka, D.A. Determination of molecular orientations on surfaces from the angular dependence of near-edge x-ray-absorption fine-structure spectra. Phys. Rev. B. 1987, 36, 7891-7905.

(36) Phillips, J. C.; Braun, R.; Wang, W.; Gumbart, J.; Tajkhorshid, E.; Villa, E.; Chipot, C.; Skeel, R. D.; Kale, L.; Schulten, K. Scalable molecular dynamics with NAMD. J. Comput. Chem. 2005, 26, 1781-1802.

(37) Vanommeslaeghe, K.; Hatcher, E.; Acharya, C.; Kundu, S.; Zhong, S.; Shim, J.; Darian, E.; Guvench, O.; Lopes, P.; Vorobyov, I.; MacKerell Jr., A.D. CHARMM general force field: A force field for drug-like molecules compatible with the CHARMM all-atom additive biological force fields. J. Comput. Chem. 2010, 31, 671690.

(38) Heinz, H.; Vaia, R. A.; Farmer, B.L.; Naik, R.R. Accurate simulation of surfaces and interfaces of Face-Centered cubic metals using 12-6 and 9-6 Lennard-Jones potentials. J. Phys. Chem. C 2008, 112, 17281-17290.

(39) Weckesser, J.; Barth, J. V; Kern K. Direct observation of surface diffusion of large organic molecules at metal surfaces: PVBA on $\mathrm{Pd}(110)$. J. Chem. Phys. 1999, 110, $5351-5354$.

(40) Humphrey, W.; Dalke, A.; Schulten, K. VMD - Visual Molecular Dynamics. J. Molec. Graphics, 1996, 14, 33-38.

(41) Booth, T.; Whanon, D.; Waine, I.W. Is chiral recognition a three-point process? Chirality 1997, 9, 96-98. 
TOC Graphic

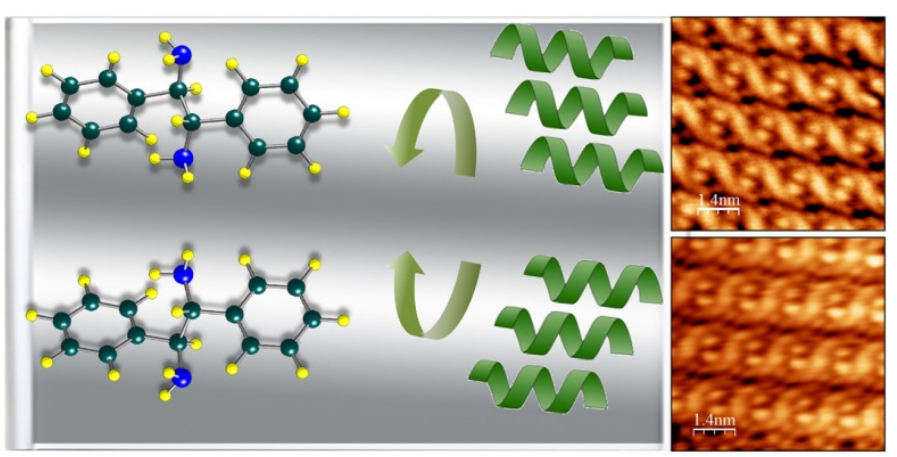




\section{Enantiopure Supramolecular Motifs of Self-Assembled Diamine-Based Chiral Molecules on $\mathrm{Cu}(\mathbf{1 0 0})$}

Rogger Palacios-Rivera, ${ }^{1}$ Esther Barrena, ${ }^{1}$ Jordi Faraudo, ${ }^{1}$ Pierluigi Gargiani, ${ }^{2}$ Miguel Angel Niño, ${ }^{3}$ Dimitri Arvanitis, ${ }^{4}$ Iwona Kowalik, ${ }^{5}$ Juan José de Miguel ${ }^{6}$ and Carmen Ocal $^{1}$

${ }^{1}$ Institut de Ciència de Materials de Barcelona (ICMAB-CSIC), Campus UAB, Bellaterra, E-08193, Barcelona, Spain.

${ }^{2}$ ALBA Synchrotron Light Source, Cerdanyola del Vallès, E-08290, Barcelona, Spain.

${ }^{3}$ Instituto Madrileño de Estudios Avanzados en Nanociencia (IMDEA-Nanociencia), C/ Faraday, 9. Cantoblanco 28049, Madrid, Spain.

${ }^{4}$ Department of Physics and Astronomy, Uppsala University, 75237-Uppsala, Sweden ${ }^{5}$ Institute of Physics, Polish Academy of Sciences, 02668-Warsaw, Poland ${ }^{6}$ Departamento Física de la Materia Condensada, Condensed Matter Physics Center (IFIMAC) and Instituto de Física de Materiales "Nicolás Cabrera”, Universidad Autónoma de Madrid, Cantoblanco 28049, Madrid, Spain. 

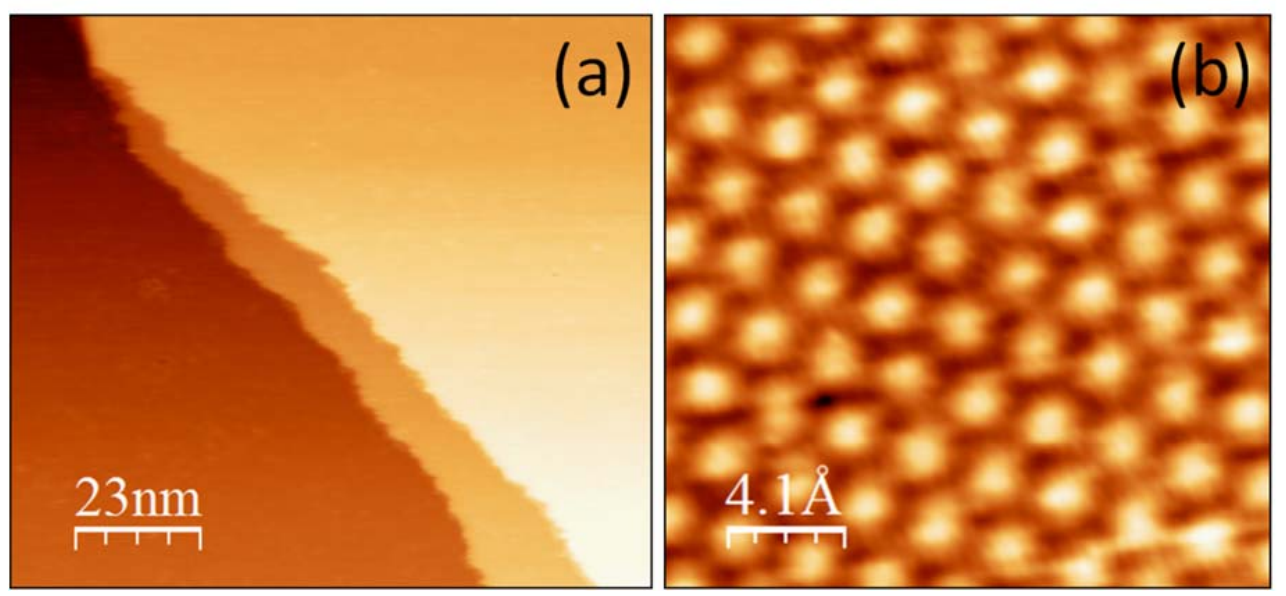

Figure S1. The instrument was calibrated by using atomic resolution images of clean single crystals as $\mathrm{Cu}(111), \mathrm{Au}(111)$ or $\mathrm{Si}(111) 7 \times 7$. In particular, here we show large scale (a) and atomic scale (b) STM images of $\mathrm{Cu}(111)$ used for $\mathrm{Z}$ and X,Y piezo calibration, respectively, via monoatomic step height and surface unit cell. STM parameters: (a) $\mathrm{I}=376 \mathrm{pA}$, bias $=-1.4 \mathrm{~V}$ and (b) $\mathrm{I}=240 \mathrm{pA}$, bias $=-0.32 \mathrm{~V}$.

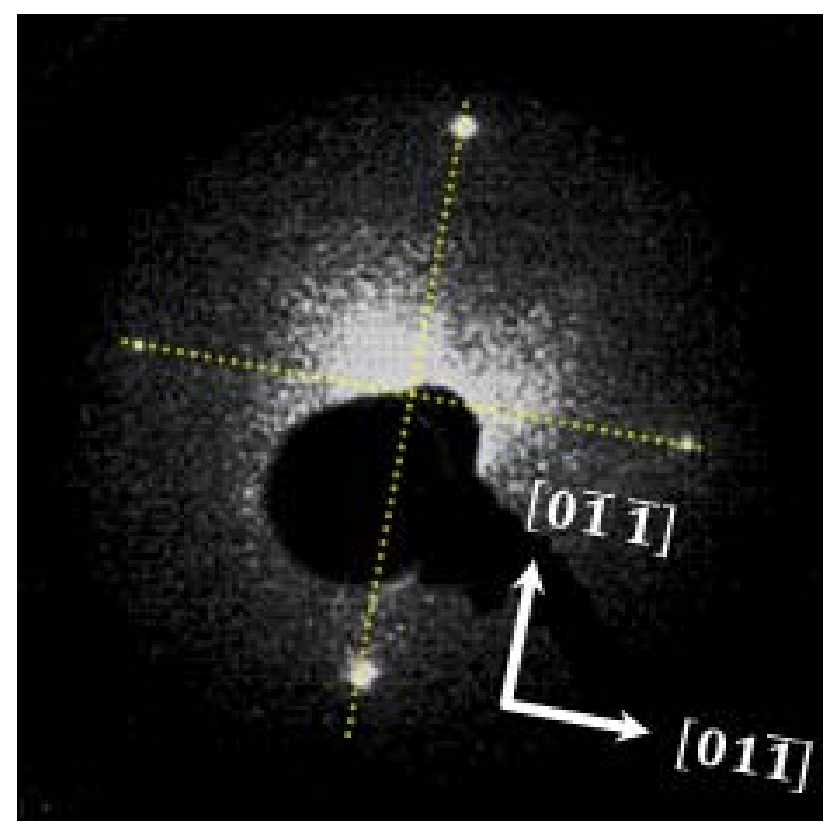

Figure S2. LEED pattern of the clean $\mathrm{Cu}(100)$ substrate was used to establish the crystal azimuth orientation that serves as reference for determining the lattice orientation of the different 2D structures reported in this work. LEED parameters: Energy: $93.4 \mathrm{eV}$, beam current: $1.8 \mu \mathrm{A}$, anode current: $0.16 \mathrm{~mA}$, cathode current: $2.40 \mathrm{~A}$, screen voltage: $6.5 \mathrm{kV}$. 


\section{Molecular Dynamics simulations methods}

Molecular Dynamics (MD) simulations consist of solving numerically the Newton equations of motion for an atomistic model of the considered system under the appropriate thermodynamic conditions. Here, we performed MD simulations using the NAMD 2.11 software $^{1}$ with a time step of 2 fs. Temperature was maintained constant at $298 \mathrm{~K}$ using a Langevin thermostat (relaxation time 1 ps).

Our simulation system consists of a single DPEDA molecule (32 atoms) and $2400 \mathrm{Cu}$ atoms organized in 12 atomic layers with 200 atoms each (area of the surface $\approx 13.11$ $\mathrm{nm}^{2}$ ). The DPEDA molecule is modelled using the CHARMM General Force Field for small organic molecules. ${ }^{2}$ The interactions of the molecules with $\mathrm{Cu}$ and the interactions between $\mathrm{Cu}$ atoms are described by the force field developed by Heinz. ${ }^{3}$

In the calculation of interactions during the simulations, we employed standard NAMD settings such as the PME method for electrostatic interactions with $1 \AA$ resolution (updated each 2 time steps) and truncation of Lennard-Jones interactions at $1.2 \mathrm{~nm}$ employing a switching function starting at $1.0 \mathrm{~nm}$. Periodic boundary conditions were employed in all directions. We performed two different simulations, one with a single molecule adsorbed at the substrate and another simulation with a high coverage of the surface.

The protocol for the first simulation was the following. We first obtained the minimum energy configuration of the molecule with NAMD. The molecule was then placed near $(1 \mathrm{~nm})$ a pre-assembled $\mathrm{Cu}(100)$ surface and a MD simulation at $298 \mathrm{~K}$ was performed during 45 ns. During this run, the molecule adsorbs at the surface and explores it during enough time to ensure equilibration. A subsequent run of 80 ns was performed to analyze in detail diffusion of the molecule at the surface. The trajectory of the molecule was Brownian, as illustrated in Figure S3a. The diffusion coefficient was calculated by fitting the root mean square displacement (rmsd) over the surface to the Einstein relation (Figure S3b). For the calculation of the adsorption free energy, we have employed the adaptive biasing force method (ABF) as implemented in NAMD. ${ }^{4}$ In these biased MD-ABF simulations, the DPEDA molecule is forced to leave the surface, sampling different values of the molecule-surface separation while all remaining degrees of freedom (motion of $\mathrm{Cu}$ atoms, molecular configuration) are thermalized. In the MD-ABF simulations performed here the generalized collective coordinate was the distance between the center of mass of the molecule and the surface which was sampled with a $0.1 \AA$ resolution during 24 ns. The force constant employed to bias the molecule position was $150 \mathrm{kcal} / \mathrm{mol} / \AA^{2}$.

In the second simulation, we started with 60 DPEDA molecules in the gas phase and an empty substrate made of $9600 \mathrm{Cu}$ atoms organized in 12 layers of 800 atoms each (area of the surface $52.43 \mathrm{~nm}^{2}$ ). We followed a protocol analogous to that of the previous case. We first performed an energy minimization and after that, a MD run at 298K. All 
the molecules initially in the gas phase adsorb, leading to a surface coverage of 1.14 molecules $/ \mathrm{nm}^{2}$. The dynamics of the molecules at the surface with this high coverage is slow, so we allowed a large simulation run until no changes in the structure of the adsorbed layer were observed. The MD run had in total 282 ns.

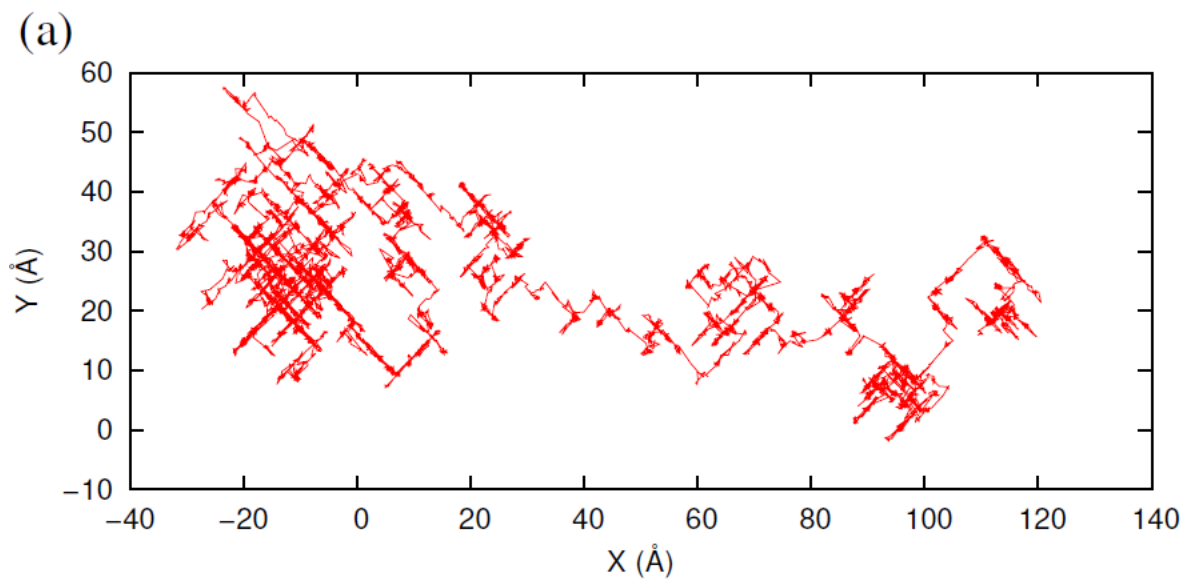

(b)

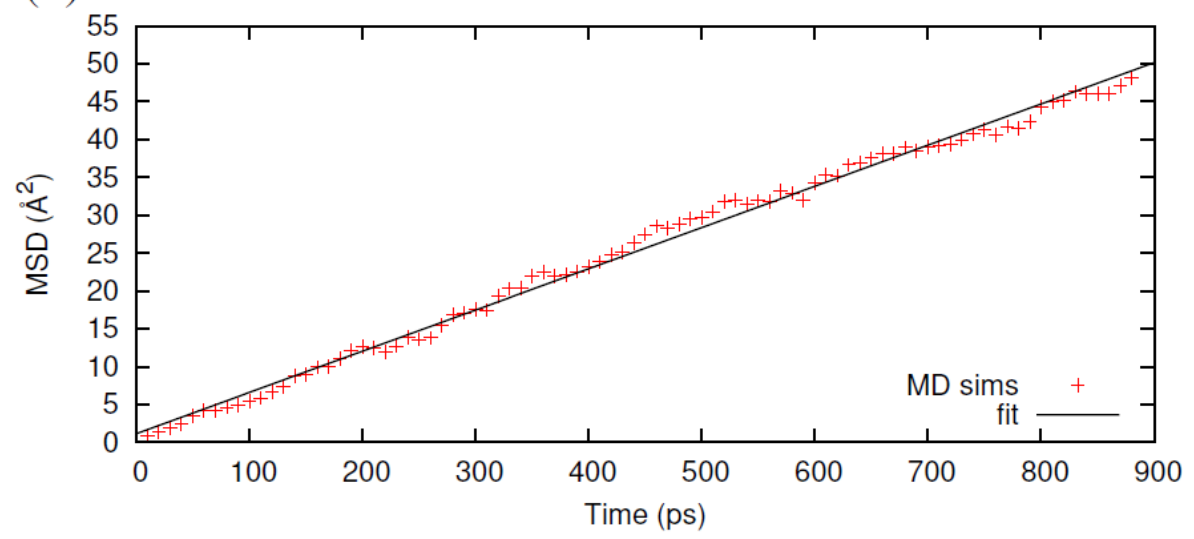

Figure S3. (a) Plot of the trajectory of the molecule over the surface in the plane of the surface as a function of time. The $(\mathrm{x}, \mathrm{y})$ coordinates over the surface are plotted each 1 ps over 120 ns of a MD trajectory. (b) Plot of the rmsd of the 120 ns trajectory shown in panel (a) as a function of time calculated from block averages. The solid line is a fit of the data to the Einstein relation rmsd $=4 D t$ (where $\mathrm{D}$ is the diffusion coefficient and $t$ is time). 


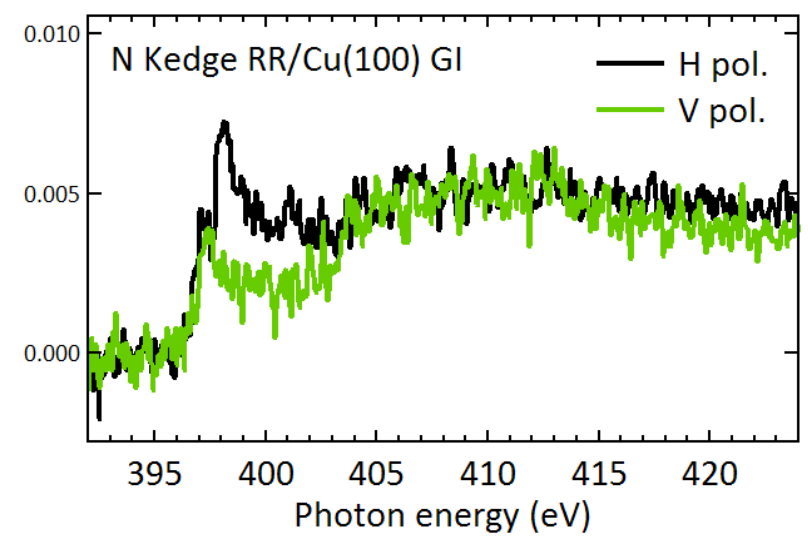

Figure S4. Nitrogen K-edge absorption intensity for linear horizontal and linear vertical incoming light polarization measured at an incidence angle of $70^{\circ}$ for (R,R)-DPEDA adsorbed on $\mathrm{Cu}(100)$.
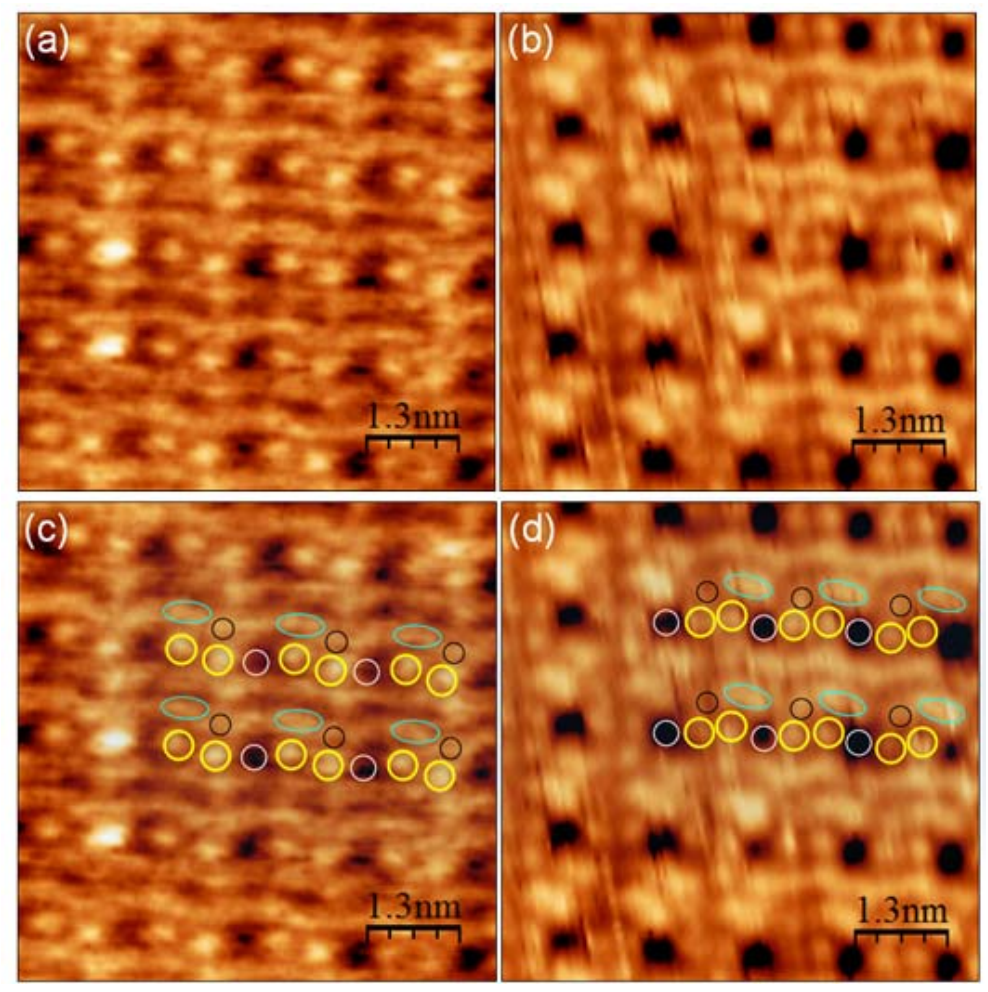

Figure S5. (a,c) and (b,d) are STM images for the A-SQ structure of (S,S)-DPEDA and $(\mathrm{R}, \mathrm{R})-\mathrm{DPEDA}$, respectively. The intrinsic chirality of the molecules, and the preservation of their chiral centers upon adsorption, is manifested within the unit cell thanks to submolecular details reflecting the sense of handedness. In order to make it clearer, mirror-like features with respect to the $[\mathbf{0} \overline{\mathbf{1}} \overline{\mathbf{1}}]$ are signaled by circles of the same color for one and the other enantiomer in the bottom images. STM parameters: (a,c) $\mathrm{I}=199 \mathrm{pA}$, bias $=+1.5 \mathrm{~V}$, (b.d) $\mathrm{I}=200 \mathrm{pA}$, bias $=+1.7 \mathrm{~V}$. 

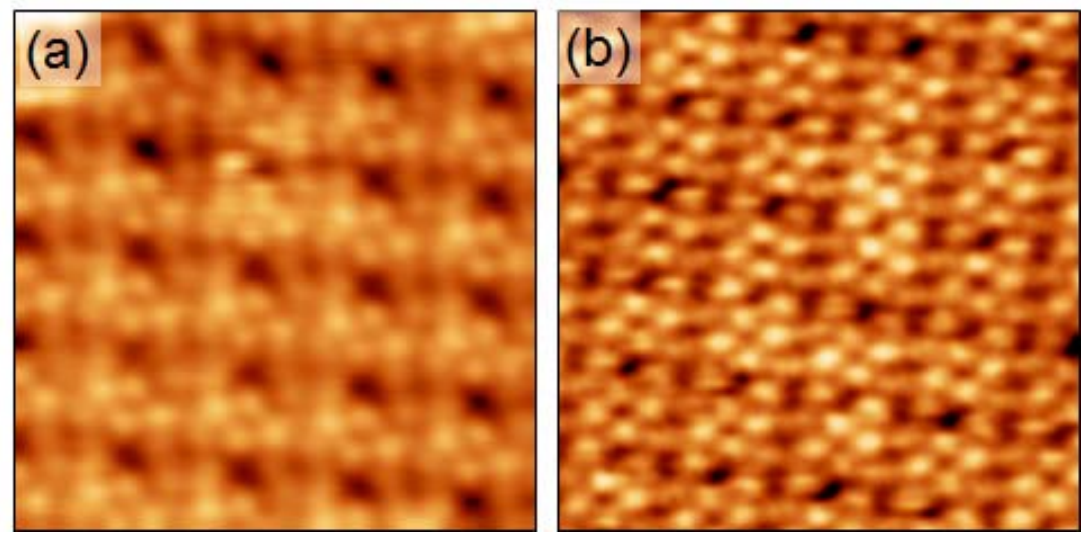

Figure S6. (a) and (b) are STM images for the A-SQ structure of (S,S)-DPEDA and (R,R)-DPEDA, respectively, obtained for different tip conditions that images shown in Figure S5. In this particular case, up to 9-10 protrusions per u.c. can be identified. STM parameters: (a) $\mathrm{I}=200 \mathrm{pA}$, bias $=+2.0 \mathrm{~V}$, (b) $\mathrm{I}=70 \mathrm{pA}$, bias $=+1.2 \mathrm{~V}$.
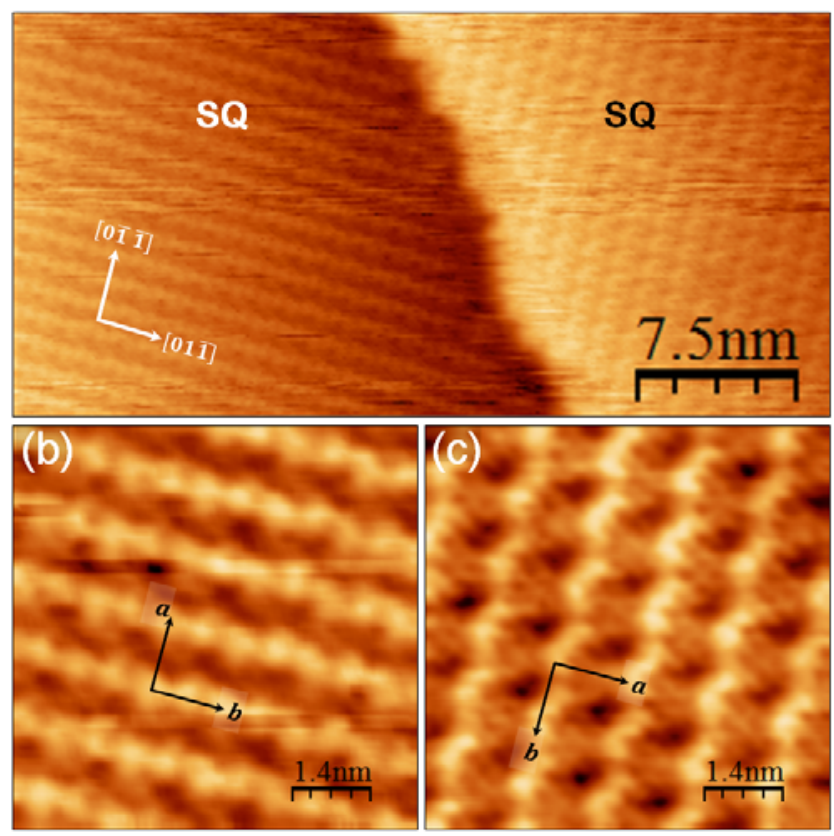

Figure S7. (a) STM image for the A-SQ structure of (S,S)-DPEDA showing equivalent domains rotated $90^{\circ}$ on neighboring terraces separated by a monoatomic substrate step. (b-c) Magnification images of each domain. STM parameters: $\mathrm{I}=164 \mathrm{pA}$ and bias $=$ $+1.80 \mathrm{~V}$. 


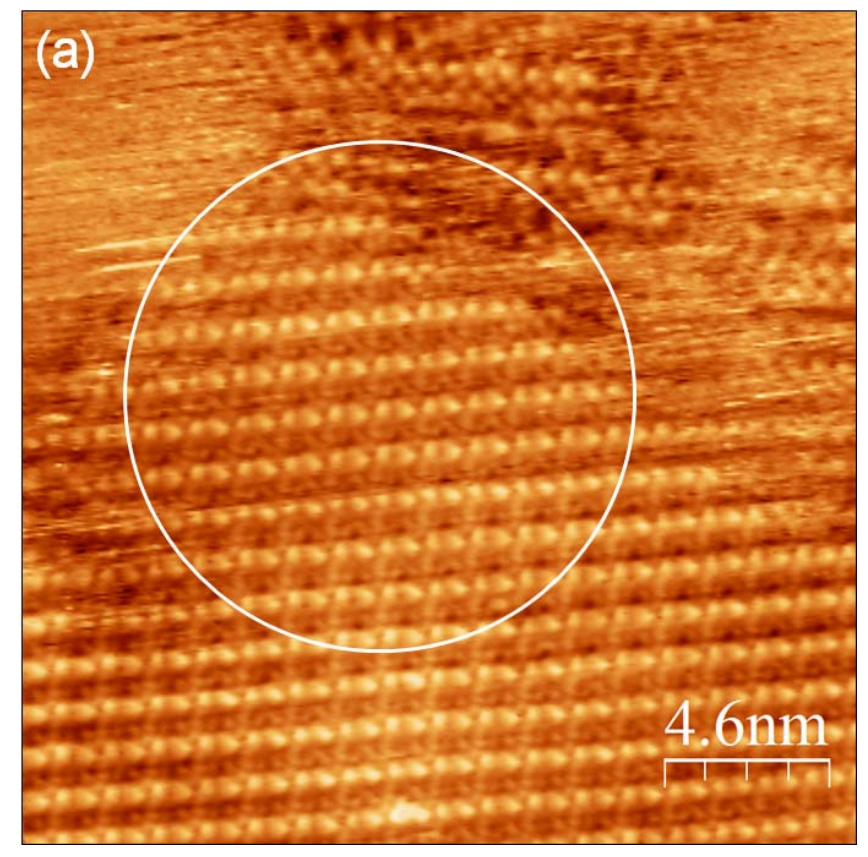

Figure S8. Large scale STM image of (R,R)-DPEDA. The encircled region serves to recognize the pattern of the SQ structure seen in the small scale image shown in Figure $5 \mathrm{~b}$ in the manuscript. STM parameters: $\mathrm{I}=105 \mathrm{pA}$, bias $=-1.1 \mathrm{~V}$.
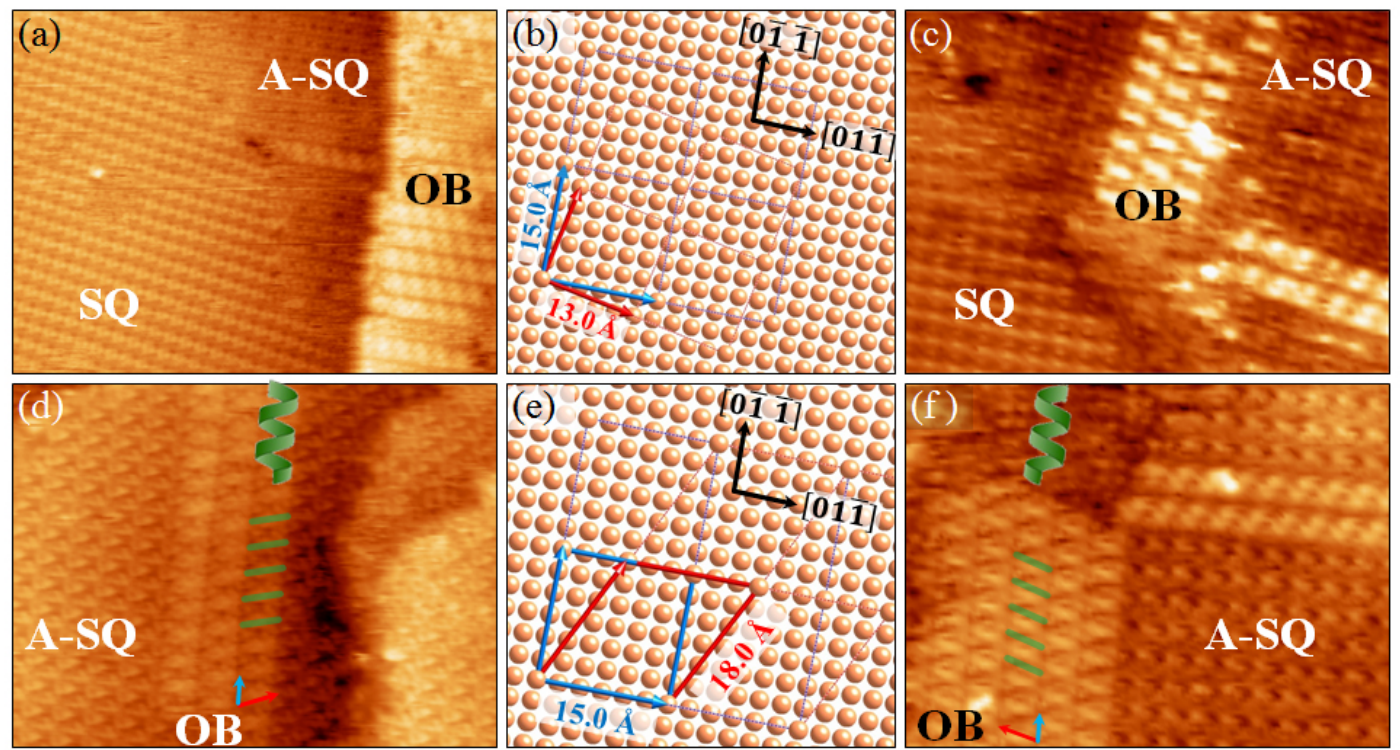

Figure S9. (a,d) and (c,f) STM images of (S,S)-DPEDA and (R,R)-DPEDA, respectively, showing coexistence of the different structures described in the manuscript: A-SQ, SQ (rotated $11^{\circ}$ ) and OB. The corresponding unit cell vectors for ASQ and SQ (b) and A-SQ and OB (e) are shown in blue and red. Note that the second layer only grows on top of the A-SQ structure in the form of stripes with a clear chiral

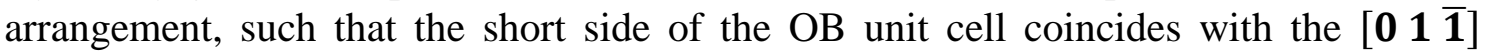
direction of the $\mathrm{Cu}(100)$. An helical design (green) has been superimposed in (d) and (f) to emphasize the helical right or left sense of the OB for each enantiomer. Images size: (a) $37 \mathrm{~nm} \times 29 \mathrm{~nm}$, (c) $17 \mathrm{~nm} \times$ x 6nm, (d) and (f) $26 \mathrm{~nm} \times 18 \mathrm{~nm}$. STM parameters: (a-d) I = $200 \mathrm{pA}$ and bias $=+2.00 \mathrm{~V}$, (c-f) $\mathrm{I}=133 \mathrm{pA}$ and bias $=-0.70 \mathrm{~V}$. 


\section{REFERENCES}

${ }^{1}$ Phillips, J. C.; Braun, R.; Wang, W.; Gumbart, J.; Tajkhorshid, E.; Villa, E.; Chipot, C.; Skeel, R. D.; Kale, L.; Schulten, K. J. Comput. Chem. 2005, 26, 1781-1802.

${ }^{2}$ Vanommeslaeghe, K.; Hatcher, E.; Acharya, C.; Kundu, S.; Zhong, S.; Shim, J.; Darian, E.; Guvench, O.; Lopes, P.; Vorobyov, I.; MacKerell Jr. A. D. J. Comput. Chem. 2010, 31, 671-690.

${ }^{3}$ Heinz, H.; Vaia, R. A.; Farmer, B. L.; Naik. R. R. J. Phys. Chem. C 2008, 112, 17281-17290.

${ }^{4}$ Hénin, J.; Fiorin, G.; Chipot, C.; Klein, M.L. J. Chem. Theory Comput., 2009, 6, 3547. 